\title{
PERFIL DOS FRAGMENTOS DE MATA ATLÂNTICA COM REGISTROS DO MUTUM-DO- -SUDESTE
}

\section{PROFILE OF ATLANTIC FOREST REMNANTS WITH RED-BILLED CURASSOW RECORDS}

\author{
Elaine Rios ${ }^{1}$ Poliane Farias Santos ${ }^{1}$ Christine Steiner São Bernardo ${ }^{2}$
}

\begin{abstract}
RESUMO
Avaliar os efeitos das mudanças no uso da terra sobre a biodiversidade se tornou uma questão-chave para conservação das espécies. Para isso, é necessário analisar a configuração e composição da paisagem em que determinada espécie ocorre. O objetivo foi traçar um perfil dos remanescentes florestais de Mata Atlântica localizados no sul da Bahia que apresentam registros atuais e históricos de Crax blumenbachii. Analisaram-se quatorze áreas utilizando diferentes escalas espaciais (escala de mancha, $500 \mathrm{~m}, 1$ e $2 \mathrm{~km}$ ) para calcular a quilometragem total e a distância mais próxima de estradas e rios até a borda da mancha, porcentagem de área ocupada por: floresta, floresta com cabrucas, pastagem e agricultura; densidade de fragmentos e a proximidade desses fragmentos até a borda de cada mancha, a área ocupada por casas e a distância dessas até a borda da mancha. Selecionou-se a escala de $2 \mathrm{~km}$ por melhor descrever a configuração e composição da paisagem. Há dois perfis de áreas com registros de mutum: (1) áreas com paisagens mais florestadas, com mais rios e estradas, pouca pastagem/agricultura e casas próximas, e (2) áreas distantes de casas, com estradas e fragmentos florestais próximos. Estas são caracteristicas de áreas com registros recentes ou históricos de mutum, e não necessariamente refletem qualidade de habitat para a espécie.
\end{abstract}

Palavras-chave: Crax blumenbachii; escala espacial; ecologia da paisagem; composição.

\begin{abstract}
Assessing the effects of land use change on biodiversity has become a key issue for species conservation. Thus, it is necessary to analyze the configuration and composition of the landscape where a species occurs. The objective was to draw a profile of the forest remnants of the Atlantic Forest located in the south of Bahia state that present current and historical records of Crax blumenbachii. We analyzed fourteen areas using different spatial scales (patch scale, $500 \mathrm{~m}, 1$ and $2 \mathrm{~km}$ ) to calculate the total distance and distance from roads and rivers to the edge of the patch, percentage of area occupied by: forest, forest with cabrucas, cabrucas, pasture and agriculture; density of fragments and the proximity of these fragments to the edge of each patch, the area occupied by houses and their distance to the edge of the patch. We selected the $2 \mathrm{~km}$ scale to best describe the configuration and composition of the landscape. There are two profiles of areas with records of red-billed curassows: (1) areas with more forested landscapes, rivers and roads, low pasture/agriculture, and near human settlements, and (2) areas distant from houses, near roads and forest fragments. These features are related to areas with recent or historic records of red-billed curassows, and do not necessarily reflect habitat quality for the species.
\end{abstract}

Keywords: Crax blumenbachii; spatial scale; landscape ecology; composition.

1 Bióloga, MSc., Programa de Pós-Graduação em Genética, Biodiversidade e Conservação, Universidade Estadual do Sudoeste da Bahia, Av. José Moreira Sobrinho, s/n, Jequiezinho, CEP 45205-490, Jequié (BA), Brasil. lanerios17@hotmail.com / polly-farias@hotmail.com

2 Bióloga, Dr ${ }^{\text {. }}$, Docente do Programa de Pós-Graduação em Genética, Biodiversidade e Conservação, Universidade Estadual do Sudoeste da Bahia, Av. José Moreira Sobrinho, s/n, Jequiezinho, CEP 45205-490, Jequié (BA), Brasil. christinesteiner@yahoo.com

Recebido para publicação em 1/05/2017 e aceito em 9/11/2017

Ci. Fl., v. 28, n. 4, out. - dez., 2018 


\section{INTRODUÇ̃̃O}

Avaliar os efeitos das mudanças no uso da terra sobre a biodiversidade se tornou uma questão-chave para conservação das espécies (SALA et al., 2000). Para tal, é necessário analisar a configuração e composição da paisagem em que determinada espécie ocorre. A intensificação das perturbações humanas na paisagem reduz o tamanho das manchas de habitat de espécies florestais e aumenta o isolamento entre estas manchas (FAHRIG, 2003). Além disso, a redução da cobertura florestal leva à homogeneização da estrutura da floresta (ROCHA-SANTOS et al., 2016). Abaixo de um determinado limiar, essa redução pode diminuir a resiliência de espécies vegetais, trazendo consequências mais severas como a perda de espécies, que coloca em risco a conservação da biodiversidade (PARDINI et al., 2010; ROCHA-SANTOS et al., 2016). Outras mudanças estão relacionadas com o efeito de borda causado pela fragmentação que direciona as florestas aos estágios iniciais de sucessão, favorecendo a proliferação de espécies pioneiras e prejudicando espécies que compõem a floresta madura como plantas lenhosas (TABARELLI; LOPES; PERES, 2008). $\mathrm{O}$ aumento na mortalidade ou ausência dessas espécies que compõem as florestas maduras tende a reduzir a qualidade do habitat, alterando a oferta e qualidade de recursos alimentares (ARROYO-RODRÍGUEZ; MANDUJANO, 2006).

Essas mudanças são uma ameaça à biota, pois alteram a composição de espécies (TABARELLI et al., 2010). A resposta de cada espécie é distinta, de modo que espécies de hábito generalista são mais resilientes, enquanto as especialistas de habitat ficam mais susceptíveis a um declínio populacional (LAURANCE, 1991; BENDER; CONTRERAS; FAHRIG, 1998). Dentre as espécies suscetíveis à perturbação antrópica, animais de médio e grande porte estão entre os mais vulneráveis à extinção, pois requerem grandes áreas de uso para sobreviver e são alvo de caçadores (CHIARELLO, 2000; PERES, 2000).

O declínio populacional de aves e mamíferos de médio e grande porte geralmente está associado a diversos mecanismos que agem em conjunto. Por exemplo, a extração de madeira (BICKNELL; PERES, 2010), redução da cobertura florestal, densidade da população humana (URQUIZA-HAAS; PERES; DOLMAN, 2009) e proximidade de estradas (VANTHOMME et al., 2013) podem facilitar e intensificar a pressão de caça (CHIARELLO, 2000; KATTAN; MUÑOZ; KIKUCHI, 2016). Esses fatores se mostraram relacionados negativamente com a abundância e permanência desses grupos em ambientes florestais. A ausência dessas espécies tende a comprometer o processo de recrutamento de plântulas e regeneração da floresta, pois muitas delas atuam como dispersoras e predadoras de sementes (PERES; PALÁCIOS, 2007; BOUFFARD; BROOKS, 2014).

Dentre essas espécies susceptíveis a ações antrópicas está o mutum-do-sudeste (Crax blumenbachii), uma ave frugívora de grande porte endêmica da Mata Atlântica e um dos poucos animais que ainda ocorre nesta região capaz de engolir e dispersar sementes grandes (IBAMA, 2004). Considerado um dos cracídeos com maior vulnerabilidade à extinção neste bioma (IBAMA, 2004; BIRDLIFE INTERNATIONAL, 2016) é classificado como criticamente em perigo em nível nacional (BRASIL, 2014), e em perigo em nível global (BIRDLIFE INTERNATIONAL, 2016). Dentre as principais ameaças a sua sobrevivência estão a caça ilegal, o isolamento e redução do tamanho das populações remanescentes, limitadas a uma pequena faixa de sua distribuição original (IBAMA, 2004; SILVEIRA; SOARES; BIANCHI, 2008). A maioria dessas populações remanescentes encontra-se em não mais que 10 fragmentos florestais na região sul do estado da Bahia (IBAMA, 2004; ALVAREZ; DEVELEY, 2010), que estão inseridos na segunda melhor região de Mata Atlântica. Esta região mantém uma parcela significativa de floresta com 17,7\% da vegetação original (RIBEIRO et al., 2009). No entanto, apenas 4,2\% da floresta remanescente está sob proteção legal, sendo a região sul da Bahia uma área prioritária para conservação da diversidade biológica e restauração das conexões florestais (RIBEIRO et al., 2009).

Nosso objetivo foi analisar a configuração e a composição da paisagem para traçar um perfil dos remanescentes florestais de Mata Atlântica situados na região sul da Bahia que apresentam registros atuais e históricos de Crax blumenbachii. 


\section{MATERIAL E MÉTODOS}

\section{Áreas de estudo}

Coletaram-se os dados em quatorze remanescentes da Mata Atlântica que diferem em tamanho e grau de proteção situadas no sul da Bahia, Brasil (Figura 1 e Tabela 1). O tipo predominante de vegetação é a floresta ombrófila densa, em diferentes estágios sucessionais, que é caracterizada por possuir altas temperaturas e elevada precipitação, bem distribuídas ao longo do ano (IBGE, 2012). Adicionalmente, outros tipos de ecossistemas naturais como manguezais, restingas e também áreas com cultivo de cacau, seringueiras, eucalipto e outros sistemas agroflorestais compõem essas áreas.

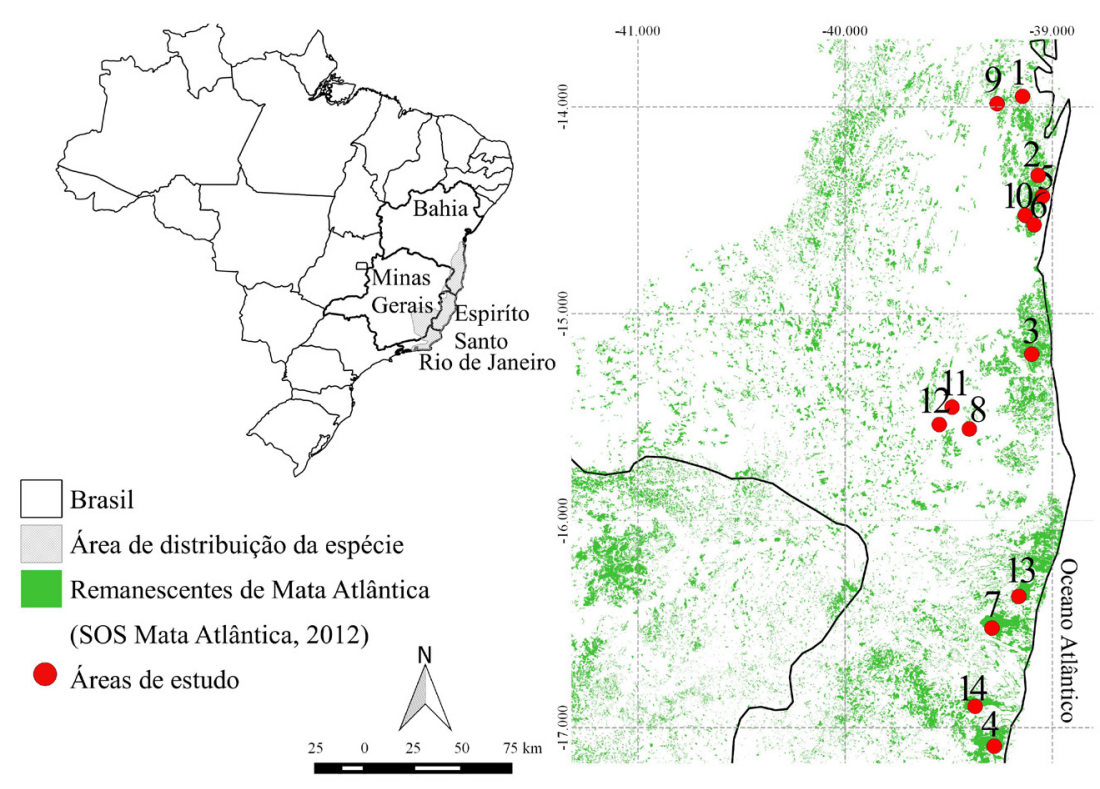

FIGURA 1: Localização dos remanescentes da Mata Atlântica analisados no presente estudo. Os números no mapa correspondem a cada área de estudo descrita na Tabela 1, campo ID.

FIGURE 1: Location of the Atlantic Forest remnants analyzed in the current study. The numbers on the map correspond to each study area described in Table 1, field ID.

As áreas foram selecionadas a partir de informações disponíveis no Plano de Ação de Crax blumenbachii (IBAMA, 2004) e registros cedidos por pesquisadores que realizaram trabalhos com o método de transectos lineares e/ou armadilhas fotográficas (ALVAREZ; DEVELEY, 2010; FLESHER; LAUFER, 2013; SOUZA et al., 2013; FERREIRA, 2014; ROCHA, 2014; BERNARDO; CANALE, 2015). Realizaram-se também entrevistas semiestruturadas com funcionários, pesquisadores e moradores que vivem dentro ou no entorno das áreas analisadas, para complementar a informação sobre ocorrência atual da espécie no local (ver material suplementar). Foram realizadas de 2 a 4 entrevistas/área, totalizando 42 entrevistas, tendo sido considerado apenas pessoas que frequentavam a mata atualmente e que não fizessem parte da mesma família. Utilizou-se uma prancha contendo imagens da fêmea e macho de Crax blumenbachii e de outros cracídeos incluindo os que não ocorrem no sul da Bahia. Este é um mecanismo utilizado para confirmar o conhecimento dos entrevistados sobre o mutum-do-sudeste, e assim evitar falsos-positivos, i.e., quando o entrevistado confirma a presença, mas não identifica corretamente a imagem da espécie na prancha e/ou quando ele confirma a presença de espécies que não ocorrem na região sul da Bahia. 
TABELA 1: Características das áreas de estudo no sul da Bahia, Brasil.

TABLE 1: Characteristics of the study areas in southern Bahia state, Brazil.

\begin{tabular}{|c|c|c|c|c|c|c|}
\hline ID & Áreas & $\begin{array}{l}\text { Estado de } \\
\text { proteção }\end{array}$ & $\begin{array}{l}\text { Ano de } \\
\text { criação }\end{array}$ & $\begin{array}{l}\text { Tamanho da } \\
\text { área }\left(\mathrm{km}^{2}\right)\end{array}$ & $\begin{array}{l}\text { Altitude } \\
(\mathrm{m})\end{array}$ & Referência \\
\hline 1 & RE Michelin & Área particular & 1984 & 0,03 & $92-338$ & Flesher e Laufer (2013) \\
\hline 2 & Capitão & $\begin{array}{c}\text { Uso } \\
\text { sustentável }\end{array}$ & 2005 & 6,6 & $100-200$ & \\
\hline 3 & Rebio de Una & $\begin{array}{l}\text { Proteção } \\
\text { integral }\end{array}$ & 1980 & 187,15 & $100-200$ & IBAMA (1997) \\
\hline 4 & $\begin{array}{c}\text { PARNA } \\
\text { Descobrimento }\end{array}$ & $\begin{array}{l}\text { Proteção } \\
\text { integral }\end{array}$ & 1999 & 226,939 & 100 & $\begin{array}{c}\text { Instituto Chico Mendes de } \\
\text { Conservação da Biodiversidade } \\
\text { (2014) }\end{array}$ \\
\hline 5 & Barra do Tijuípe & $\begin{array}{c}\text { Uso } \\
\text { sustentável }\end{array}$ & 1993 & 1,87 & $200-300$ & $\begin{array}{l}\text { V\&C Engenheiros Consultores } \\
\text { (1998); Bernardo e Canale } \\
\text { (2015) }\end{array}$ \\
\hline 6 & Sul do PESC & $\begin{array}{l}\text { Proteção } \\
\text { integral }\end{array}$ & 1997 & 0,027 & $200-300$ & Bahia (2005) \\
\hline 7 & PARNA Pau-Brasil & $\begin{array}{l}\text { Proteção } \\
\text { integral }\end{array}$ & 1999 & 0,115 & 100 & Wikiparques (2016) \\
\hline 8 & $\begin{array}{l}\text { Faz. Nossa Senhora } \\
\text { Auxiliadora }\end{array}$ & Área particular & - & 0,01 & $200-400$ & Climate-data.org (2016) \\
\hline 9 & Faz. Vale do Juliana & Área particular & - & 0,048 & $200-300$ & $\begin{array}{c}\text { Fazendas Reunidas Vale do } \\
\text { Juliana (2016) }\end{array}$ \\
\hline 10 & Sede do PESC & $\begin{array}{l}\text { Proteção } \\
\text { integral }\end{array}$ & 1997 & 0,02 & $0-200$ & \\
\hline 11 & Camacan & Área particular & - & 2,47 & $200-950$ & Climate-data.org (2016) \\
\hline 12 & Serra Bonita & $\begin{array}{c}\text { Uso } \\
\text { sustentável }\end{array}$ & 1997 & 20 & $180-960$ & Martins e Galileo (2010) \\
\hline 13 & Estação Veracel & $\begin{array}{c}\text { Uso } \\
\text { sustentável }\end{array}$ & 1998 & 0,06 & Oct-80 & Veracel (2007) \\
\hline 14 & $\begin{array}{l}\text { PARNA Monte } \\
\text { Pascoal }\end{array}$ & $\begin{array}{c}\text { Proteção } \\
\text { integral }\end{array}$ & 1961 & 225,006 & $95-536$ & Brasil (1995); IBAMA (2005) \\
\hline
\end{tabular}

Em que: ID = Número de identificação da área de estudo; RE = Reserva Ecológica; RPPN = Reserva Particular do Patrimônio Natural; Rebio = Reserva Biológica; PARNA = Parque Nacional; APA = Área de Proteção Ambiental; PESC $=$ Parque Estadual Serra do Conduru; Faz. $=$ Fazenda; $(-)$ sem informação.

\section{Métricas da mancha florestal e da paisagem}

Avaliaram-se diferentes variáveis considerando a área interna do polígono de mata (escala de mancha) e as áreas de influência de $2 \mathrm{~km}, 1 \mathrm{~km}$ e $500 \mathrm{~m}$ de raio (escala de paisagem). A área máxima de influência $(2 \mathrm{~km})$ foi considerada com base na distância média entre as áreas de vida dos casais de Crax blumenbachii nativos e reintroduzidos, que variou de 2 a 3 km (SICK, 1997; BERNARDO et al., 2011). Para não haver sobreposição da área de vida decidiu-se analisar a resposta da espécie numa escala $<3 \mathrm{~km}$ no entorno de cada área estudada.

Utilizou-se o software ArcGis (versão 10.3 Environmental Systems Research Institute) e Google Earth para a delimitação dos polígonos das áreas de estudo e extração de informações das variáveis 
contidas nas camadas ambientais, obtidas em diferentes fontes (LANDAU; HIRSCH; MUSINSKY, 2003; AGÊNCIA NACIONAL DE ÁGUAS, 2010; FUNDAÇÃO SOS MATA ATLÂNTICA, 2012; BRASIL, 2016; INSTITUTO CHICO MENDES DE CONSERVAÇÃO DA BIODIVERSIDADE, 2016).

Calculou-se a (1) porcentagem de área ocupada por floresta somando a porcentagem de vegetação em estágios de sucessão primária e secundária; (2) porcentagem de área ocupada por floresta e cabrucas; (3) porcentagem de área ocupada por cabrucas; (4) porcentagem de área ocupada por pastagem e agricultura; (5) densidade de fragmentos, dividindo o número de fragmentos pela área; (6) média da distância mais próxima desses fragmentos à borda da mancha; (7) quilometragem total de rodovias e estradas sem pavimentação; (8) média da distância mais próxima de rodovias, estradas sem pavimentação à borda da mancha; (9) quilometragem total de rios; (10) distância mais próxima de rios até a borda da mancha; (11) área ocupada por casas; (12) média da distância de casas até a borda da mancha: não se fez distinção entre residências e outras construções como igrejas, escolas, galpões ou pequenas cabanas próximas à mata, pois todos indicam a presença de humanos, embora possam oferecer impactos diferentes. Por fim, o (13) índice da forma (IF) de cada polígono aplicando a fórmula:

$\mathrm{IF}=(\mathrm{P} / 200)^{*}(\pi * \mathrm{~A})^{0.5}$

Em que: $\mathrm{IF}=$ índice da forma; $\mathrm{P}=$ perímetro (m); $\mathrm{A}$ = área do fragmento florestal (ha).

Assim, as áreas que obtiveram resultado $=1$ foram consideradas áreas com forma circular, enquanto as áreas com valores $>1$ foram consideradas áreas com formato mais irregular (LAURANCE, 1991; LAURANCE; YENSEN, 1991).

Não foram analisadas as variáveis relacionadas à densidade de fragmentos e distância de estradas, rios, fragmentos florestais e casas na escala de mancha, do mesmo modo que o índice da forma (IF) foi calculado apenas na escala de mancha. A porcentagem de declividade do terreno foi medida em ambas as escalas, local e de paisagem, e utilizada nas análises em um estudo piloto. Porém, esta variável não foi considerada devido à ausência de variância, que enviesava os resultados.

\section{Análise dos dados}

Para avaliar o buffer mais apropriado para traçar o perfil dos remanescentes florestais com registros de Crax blumenbachii, comparou-se o coeficiente de determinação (R2) entre cada variável resposta e a porcentagem de floresta, considerando a porcentagem de floresta como variável independente e cada métrica como variável dependente. Para tanto, utilizou-se o software BioEstat versão 5.0 (2007). Selecionou-se o buffer que apresentou os maiores valores de coeficiente de determinação $\left(\mathrm{R}^{2}\right)$ entre as métricas de paisagem e de mancha. Este procedimento foi similar a Rocha-Santos e colaboradores (2016).

Verificou-se a multicolinearidade entre as variáveis coletadas na escala de $2 \mathrm{~km}$, escala selecionada, utilizando a correlação de Spearman. Consideraram-se para análises subsequentes apenas as variáveis não autocorrelacionadas $(\mathrm{r} \leq 0,70)$. Posteriormente, realizou-se uma Análise dos Componentes Principais (ACP) para descrever o perfil dos 14 remanescentes da Mata Atlântica estudados e verificar quais os mais semelhantes. As análises estatísticas foram realizadas no software Past versão 3.10 (2013).

\section{RESULTADOS E DISCUSSÃO}

A escala de $2 \mathrm{~km}$ apresentou os maiores valores de $\mathrm{R}^{2}$ e foi escolhida para descrever a configuração e composição da paisagem (Tabela 2). As oito variáveis não autocorrelacionadas foram: quilometragem total de rios e estradas, distância de estradas até a borda do fragmento florestal, distância de casas até a borda do fragmento florestal, área total ocupada por casas, \% de floresta, \% de pastagem e agricultura e proximidade de fragmentos florestais. 
TABELA 2: Coeficiente de determinação $\left(\mathrm{R}^{2}\right)$ das variáveis da paisagem em relação à porcentagem de floresta nas diferentes escalas. Os maiores valores do coeficiente de determinação $\left(\mathrm{R}^{2}\right)$ foram destacados em negrito.

TABLE 2: Coefficient of determination $\left(\mathrm{R}^{2}\right)$ of the landscape variables related to the percentage of forest in the different scales. The highest values of coefficient of determination $\left(\mathrm{R}^{2}\right)$ were highlighted in bold.

\begin{tabular}{ccccc}
\hline Variáveis dependentes & Interior & $500 \mathrm{~m}$ & $1 \mathrm{~km}$ & $2 \mathrm{~km}$ \\
\hline Kmr & $0,364^{* *}$ & $0,484^{* *}$ & $0,462^{* *}$ & $\mathbf{0 , 6 2 3 * *}$ \\
Drb & & 0,037 & 0,004 & $\mathbf{0 , 1 4 1}$ \\
Kme & $0,400^{* *}$ & $0,531^{* *}$ & $0,488^{* *}$ & $\mathbf{0 , 5 9 4 * *}$ \\
Deb & & 0,146 & $\mathbf{0 , 1 5 5}$ & 0,126 \\
Ca & 0,064 & 0,031 & 0,213 & $\mathbf{0 , 4 3 0 * *}$ \\
Floca & $\mathbf{0 , 4 7 5 * *}$ & 0,11 & 0,246 & 0,055 \\
Pa & 0,083 & 0,056 & 0,096 & $\mathbf{0 , 2 2}$ \\
Df & - & 0,005 & 0,006 & $\mathbf{0 , 0 0 6}$ \\
Pf & - & 0,093 & $\mathbf{0 , 1 2 6}$ & 0,045 \\
Dcb & - & 0,006 & 0,193 & $\mathbf{0 , 1 9 8}$ \\
Atc & 0,054 & $\mathbf{0 , 1 1 8}$ & 0,036 & 0,023 \\
If & $0,424^{* *}$ & & & \\
\hline
\end{tabular}

Em que: $\mathrm{kmr}=$ quilometragem total de rios; $\mathrm{drb}=$ distância de rios até a borda do fragmento florestal; $\mathrm{kme}=$ quilometragem total de estradas; deb $=$ distância de estradas até a borda do fragmento florestal; $\mathrm{ca}=\%$ de cabruca; floca $=\%$ de floresta com cabruca; $\mathrm{pa}=\%$ de pastagem e agricultura; $\mathrm{df}=$ densidade de fragmentos florestais; $\mathrm{pf}=$ proximidade de fragmentos florestais; dcb = distância de casas até a borda do fragmento florestal; atc = área total ocupada por casas; if = índice da forma do fragmento florestal; **p $<0.01$.

Os dois primeiros eixos da Análise dos Componentes Principais (ACP) explicaram $\sim 65 \%$ da variância total dos dados (eixo $1=48,5 \%$ e eixo $2=16,7 \%$ ). O primeiro eixo foi relacionado positivamente com a quilometragem total de estradas (peso $=0,48$ ), quilometragem total de rios (peso $=0,48$ ) e porcentagem de área ocupada por floresta ( $\mathrm{peso}=0,41$ ), e foi relacionado negativamente com a distância de casas até a borda da mancha (peso $=-0,35$ ) e porcentagem de área ocupada por pastagem e agricultura (peso $=-0,29$ ). O segundo eixo foi relacionado positivamente com a proximidade de fragmentos até a borda da mancha (peso $=0,79)$, distância de casas até a borda da mancha $($ peso $=0,40)$, e relacionado negativamente com a distância de estradas até a borda da mancha (peso $=-0,35$ ) (Figura 2). Assim, identificaram-se dois tipos de perfis de paisagens no entorno de $2 \mathrm{~km}$ dos remanescentes florestais com registros de Crax blumenbachii: (i) áreas com alta porcentagem de cobertura florestal, com muitas estradas e muitos rios, com baixa porcentagem de pastagem/agricultura, com casas próximas aos remanescentes estudados, e.g., Parques Nacionais Monte Pascoal (14), Descobrimento (4), Pau-Brasil (7) e a Reserva Biológica de Una (3); (ii) áreas distantes de casas, com fragmentos florestais próximos e com estradas próximas, e.g., Capitão (2) (Figura 2). 


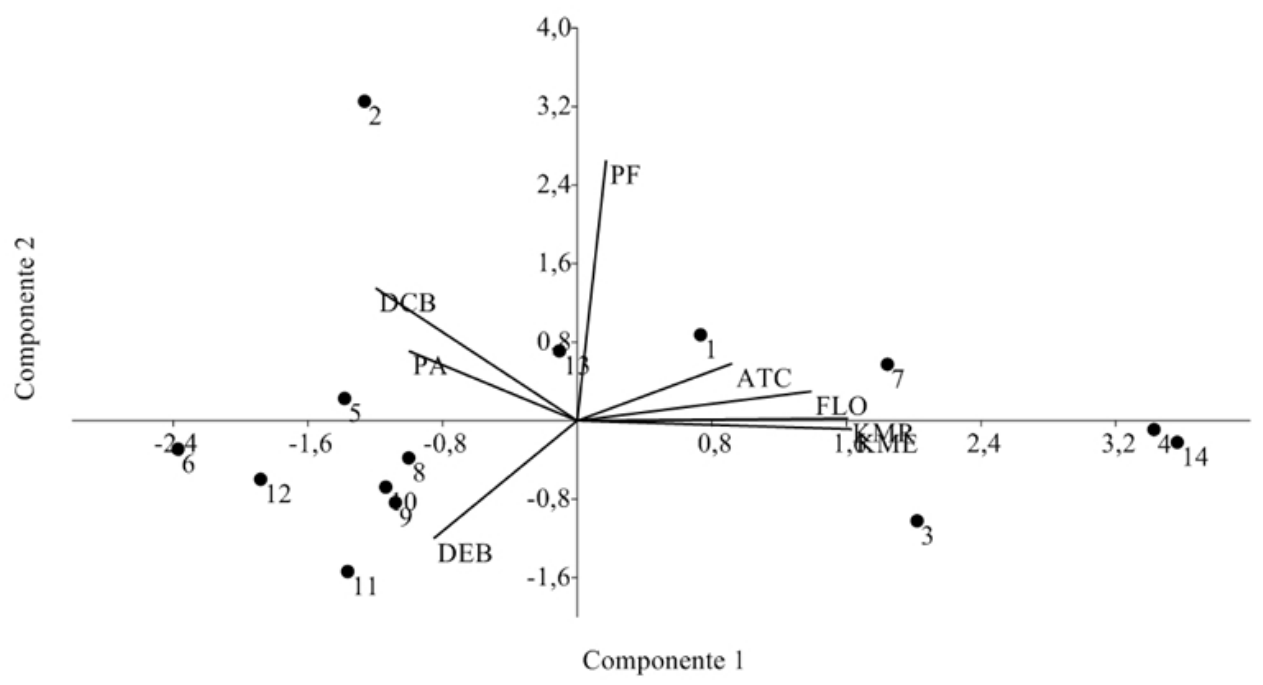

FIGURA 2: Biplot gerado a partir da Análise dos Componentes Principais (ACP) representando características da paisagem no entorno de $2 \mathrm{~km}$ de 14 fragmentos florestais de Mata Atlântica com registros atuais e históricos de Crax blumenbachii. Os números indicam as áreas de estudo (Tabela 1, campo ID).

FIGURE 2: Biplot obtained by Principal Component Analysis (PCA), representing landscape features within a $2 \mathrm{~km}$ buffer radius around 14 Atlantic Forest remnants with current and historical records of Crax blumenbachii. The numbers indicate the study areas (Table 1, ID field).

Os remanescentes florestais do Monte Pascoal, Descobrimento, Pau-Brasil e Una possuem perfil semelhante, pois apresentaram muitas estradas vicinais em seu interior e/ou no entorno de $2 \mathrm{~km}$ (Figura 2). Crax blumenbachii é frequentemente visto fazendo uso de estradas vicinais locais e trilhas dentro de fragmentos florestais em que a pressão de caça é baixa (IBAMA, 2004; SRBEK-ARAUJO; SILVEIRA; CHIARELLO, 2012; ALVES; LÓPEZ-IBORRA; SILVEIRA, 2015). Tal hábito está relacionado principalmente ao forrageio (SRBEK-ARAUJO; SILVEIRA; CHIARELLO, 2012), facilidade de deslocamento e ao comportamento de ajeitar a plumagem (COLLAR; GONZAGA, 1988). Por outro lado, estradas facilitam o acesso humano às matas, alterando a paisagem em que estão inseridas e consequentemente interferindo nas espécies que as compõem (COFFIN, 2007). Para cracídeos há relatos de aumento na pressão de caça e destruição do habitat, devido à extração seletiva de madeira, em áreas em que estradas foram abertas e/ ou pavimentadas (THIOLLAY, 1992; MARTÍNEZ-MORALES, 1999). A proximidade de casas e porcentagem de pastagem e agricultura também estão relacionadas à modificação do habitat e aumento da pressão de caça (BROOKS, 1999; HAYES; SANASIE; SAMAD, 2009; AUBAD; ARAGÓN; RODRÍGUEZ, 2010; THORNTON; BRANCH; SUNQUIST, 2012).

Os Parques Nacionais do Monte Pascoal, Descobrimento, Pau-Brasil e Rebio de Una são similares por terem apresentado maior quilometragem de rios (Figura 2). Trabalhos anteriores mostram a preferência dos cracídeos por áreas próximas a fontes de água, sendo locais usados para forrageio e construção de seus ninhos (MARTÍNEZ-MORALES, 1999; IBAMA, 2004; BODRATI; COCKLE, 2006; HILL; ARAÑIBAR-ROJAS; MACLEOD, 2008; LUNA-MAÍRA et al., 2013). Assim, uma estratégia eficaz para a conservação de mutum-do-sudeste e outros cracídeos é contemplar amplas extensões de rios no planejamento e estabelecimento de áreas protegidas (LUNA-MAÍRA et al., 2013; SCARANO; CEOTTO, 2015). Estas mesmas áreas também apresentaram maior porcentagem de cobertura florestal no entorno, enquanto a área Capitão apresentou fragmentos florestais mais próximos a sua borda (Figura 2). Essas características são importantes, pois grandes frugívoros têm maior preferência por áreas florestadas inseridas em uma paisagem pouco fragmentada (PEREIRA; DAILY; ROUGHGARDEN, 2004; BICKNELL; PERES, 2010). Áreas em que mutuns não são registrados há mais de 15 anos, como Camacan (11), estão inseridas em uma 
paisagem fragmentada. De fato, esse tipo de cenário é uma ameaça à sobrevivência da espécie (BIRDLIFE INTERNATIONAL, 2016), cujo tamanho de área de vida de um casal adulto é, em média, 250 ha (BERNARDO et al., 2011).

As áreas do Descobrimento, Pau-Brasil e Rebio de Una, nas quais Crax blumenbachii ainda persiste, estão mais relacionadas a um habitat com menor porcentagem de pastagem e agricultura e mais distantes de casas no seu entorno. No entanto, esse cenário não foi observado em todos os locais em que há registro atual da espécie, como na área Capitão. Alguns autores citam o uso de plantações pelo mutum-do-sudeste, situadas próximas a casas na zona rural onde a pressão de caça é baixa (ALVAREZ; DEVELEY, 2010; BERNARDO et al., 2011). Contudo, a frequência de forrageio em áreas próximas a casas é baixa, mesmo por espécies de cracídeos mais resilientes a um certo nível de modificação no habitat, como é o caso de Penelope superciliaris (MIKICH, 2002).

Os assentamentos humanos também podem funcionar como uma barreira física isolando populações (KATTAN et al., 2006) tornando-se um problema para manutenção da dinâmica fonte-dreno (PULLIAM, 1988) especialmente porque a recuperação das populações de cracídeos é trabalhosa quando sofrem intensa pressão de caça (BEGAZO; BODMER, 1998), aumentando o risco de extinção local. Dentre os fragmentos florestais analisados, o da Fazenda Nossa Senhora Auxiliadora fica próximo a uma área de assentamento humano. Como não possui proteção legal, é comum a prática da caça pela população local, tendo sido observado também à presença de vários cães durante uma visita na área.

\section{CONCLUSÕES}

Identificaram-se dois perfis de áreas com registros de mutum: (1) áreas com paisagens mais florestadas, com mais rios e estradas, pouca pastagem/agricultura e casas próximas, e (2) áreas distantes de casas, com estradas e fragmentos florestais próximos. Pode-se inferir que as áreas em que atualmente há registros de mutum (e.g. Una, que se encaixa no perfil 1) possuem caracteristicas importantes para a conservação da espécie. Assim, áreas com perfis semelhantes são recomendadas para a conservação do mutum. No entanto, deve-se ter em mente que os perfis referem-se a áreas em que há ou houve registros de mutum, e não necessariamente refletem qualidade ótima de habitat para a espécie.

\section{AGRADECIMENTOS}

Agradecemos o apoio financeiro da Coordenação de Aperfeiçoamento de Pessoal de Nível Superior (CAPES), ao Programa de Pós-Graduação em Genética, Biodiversidade e Conservação, aos moradores e responsáveis pelas áreas analisadas e pesquisadores, que colaboraram com informações para esse estudo.

\section{REFERÊNCIAS}

AGÊNCIA NACIONAL DE ÁGUAS (Brasil). Sistema de informações hidrológicas. Brasília: ANA, 2010. Disponível em: <hidroweb.ana.gov.br/HidroWeb.asp?TocItem=4100>. Acesso em: jan. 2016.

AlvareZ, P. F.; DEVEleY, A. D. Conservação do Mutum-do-Sudeste (Crax blumenbachii) Cinco anos de implementação do Plano de Ação. São Paulo: SAVE Brasil, 2010. 47 p.

ALVES, F.; LÓPEZ-IBORRA, G. M.; SILVEIRA, L. F. Population size assessment of the endangered redbilled curassow Crax blumenbachii: accouting for variation in detectability and sex-biased estimates. Oryx, Cambridge, v. 51, p. 137-145, 2015.

ARROYO-RODRÍGUEZ, V.; MANDUJANO, S. Forest fragmentation modifies quality for Alouatta palliata. International Journal of Primatology, New York, v. 27, n. 4, p. 1079-1096, 2006.

AUBAD, J.; ARAGÓN, P.; RODRÍGUEZ, M. A. Human access and landscape structure effects on Andean forest bird richness. Acta Oecologica, Paris, v. 36, p. 396-402, 2010.

BAHIA (Estado). Secretaria do Meio Ambiente e Recursos Hídricos. Plano de Manejo do Parque Estadual Serra do Conduru. Salvador: SEMARH, 2005. Disponível em: $<$ http://www.parquedoconduru. org/index.php/o-parque/plano-de-manejo>. Acesso em: mar. 2016. 
BEGAZO, A. J.; BODMER, R. E. Use and conservation of Cracidae (aves: Galliformes) in the peruvian amazon. Oryx, Cambridge, v. 32, p. 301-309, 1988.

BENDER, D. J.; CONTRERAS, T. A.; FAHRIG, L. Habitat loss and population decline: a meta-analysis of the patch size effect. Ecology, Durham, v. 79, n. 2, p. 517-533, 1998.

BERNARDO, C. S. S.; CANALE, G. Vertebrados terrestres de médio e grande porte da Barra do Tijuípe. [s. 1.]: Empresa de consultoria Seleção Natural, 2015.

BERNARDO, C. S. S. et al. Using post-release monitoring data to optimize avian reintroduction programs: a 2-year case study from the brazilian Atlantic rainforest. Animal Conservation, Cambridge, v. 14, p. 676686, 2011.

BICKNELL, J.; PERES, C. A. Vertebrate population responses to reduced-impact logging in a neotropical forest. Forest Ecology Management, Amsterdam, v. 259, p. 2267-2275, 2010.

BIRDLIFE INTERNATIONAL. Crax blumenbachii. The IUCN red list of threatened species. Cambridge: [s. n.], 2016. Disponível em <http://www.iucnredlist.org/details/22678544/0>. Acesso em: mar. 2016.

BIOESTAT. Aplicações estatísticas nas áreas das ciências bio-médicas. Pará: Instituto Mamiraua, 2007. BODRATI, A.; COCKLE, K. L. Habitat, distribution, and conservation of Atlantic forest birds in Argentina: notes on nine rare or threatened species. Ornitologia Neotropical, Washington, v. 17, p. 243-258, 2006.

BOUFFARD, L. A.; BROOKS, D. M. The role of the white-winged guan (Penelope albipennis) in seed dispersal and predation in tumbesian dry forest, Peru. Journal of Sustainable Forestry, [s. 1.], v. 33, p. 184-194, 2014.

BRASIL. Departamento Nacional de Infraestrutura de Transportes. [Website]. Brasília: DNIT, 2016. Disponível em: <www.dnit.gov.br/planejamento-e-pesquisa/dnit-geo/copy_of_dnitgeo>. Acesso em: jun. 2016.

BRASIL. Ministério do Meio Ambiente. Plano de ação emergencial para o Parque Nacional de Monte Pascoal. Brasília: Instituto Brasileiro do Meio Ambiente e dos Recursos Naturais Renováveis, 1995. Disponível em: $<$ http://www.icmbio.gov.br/portal/images/stories/docs-planos-de-manejo/parnah_do_monte pascoal_plano_de_acao_emergencial.pdf > . Acesso em: mar. 2017.

BRASIL. Portaria MMA n ${ }^{\circ}$ 444, de 17 de dezembro de 2014. Diário Oficial da União, Brasília, DF, 18 dez. 2014. Seção 1, p. 121-126.

BROOKS, D. M. Pipile as a protein source to rural hunters and Amerindians. In: BROOKS, D. M.; BEGAZO, A. J.; OLMOS, F. Biology and conservation of the piping guans (Pipile). Houston: Special Monograph Series, 1999. p. 42-50.

CHIARELLO, A. G. Influência da caça ilegal sobre mamíferos e aves das matas de tabuleiro do norte do estado do Espírito Santo. Boletim do Museu de Biologia Mello Leitão, Espírito Santo, v. 11/12, p. 229-247, 2000.

CLIMATE-DATA.ORG. Dados climáticos para cidades mundiais. 2016. Disponível em: <pt.climate-data.org>. Acesso em: abr. 2016.

COLLAR, N. J.; GONZAGA, L. A. P. O mutum Crax blumenbachii na Reserva Florestal Particular de Linhares - ES. Espaço, Ambiente e Planejamento, [s. 1.], v. 2, n. 8, p. 3-34, 1988.

COFFIN, A. W. From roadkill to road ecology: a review of the ecological effects of roads. Journal of Transport Geography, London, v. 15, p. 396-406, 2007.

FAHRIG, L. Effects of habitat fragmentation on biodiversity. Annual Review of Ecology, Evolution, and Systematics, Estados Unidos, v. 34, p. 487-514, 2003.

FAZENDAS REUNIDAS VALE DO JULIANA. [Website]. Ituberá: Fazendas Reunidas Vale do Juliana, 2015. Disponível em: <http://valedojuliana.com.br/wp/>. Acesso em: set. 2016.

FERREIRA, P. C. R. Estimativas populacionais e áreas prioritárias para a conservação de Callithrix kuhlii (Coimbra-Filho, 1985). 2014. 97 f. Dissertação (Mestrado em Genética, Biodiversidade e Conservação) - Universidade Estadual do Sudoeste da Bahia, Jequié, 2014.

FLESHER, K. M.; LAUFER, J. Protecting wildlife in a heavily hunted biodiversity hotspot: a case study from the Atlantic forest of Bahia, Brazil. Tropical Conservation Science, [s. 1.], v. 6, n. 2, p. 181-200, 2013.

FUNDAÇÃO SOS MATA ATLÂNTICA. SOS Mata Atlântica. São Paulo: Fundação SOS Mata Atlântica, 2012. Disponível em: <mapas.sosma.org.br>. Acesso em: fev. 2015.

HAYES, F. E.; SANASIE, B.; SAMAD, I. Status and conservation of the critically endangered Trinidad 
piping-guan Aburria pipile. Endangered Species Research, Alemanha, v. 7, p. 77-84, 2009.

HILL, D. L.; ARAÑIBAR-ROJAS, H.; MACLEOD, R. Wattled curassows in Bolivia: abundance, habitat use, and conservation status. Journal of Field Ornithology, New Ipswich, v. 79, n. 4, p. 345-351, 2008. IBAMA. Plano de ação para a conservação do mutum-do-sudeste Crax blumenbachii. 1. ed. Brasília: IBAMA, 2004. 50 p. (Série espécies ameaçadas).

IBAMA. Plano de prevenção aos incêndios florestais Parque Nacional do Monte Pascoal. Itamaraju: Ministério do Meio Ambiente, 2005. Disponível em: <http://www.ibama.gov.br/documentos/planos-operativos-em-ucs-federais>. Acesso em: mar. 2016.

IBAMA. Reserva Biológica de Una. Plano de manejo. [s. 1.]: Instituto Chico Mendes da Biodiversidade, 1997. Disponível em: <http:/www.icmbio.gov.br/portal/images/stories/imgs-unidades-coservacao/ REBIO\%20Una.pdf >. Acesso em: mar. 2016.

IBGE. Manual técnico da vegetação brasileira: sistema fitogeográfico, inventário das formações florestais e campestres, técnicas e manejo de coleções botânicas, procedimentos para mapeamentos. 2. ed. Rio de Janeiro: IBGE, 2012. 275 p.

INSTITUTO CHICO MENDES DE CONSERVAÇÃO DA BIODIVERSIDADE. Plano de Manejo Parque Nacional do Descobrimento. Brasília: Ministério do Meio Ambiente, 2014. Disponível em: $<$ http://www.icmbio.gov.br/portal/component/content/article?id=2197:parna-do-descobrimento $>$. Acesso em: mar. 2017.

INSTITUTO CHICO MENDES DE CONSERVAÇÃO DA BIODIVERSIDADE. Relação de UCS com plano de manejo. [s. 1.]: ICMBIO, 2016. Disponível em: $<$ http://www.icmbio.gov.br/portal/planosmanejo? start $=160>$. Acessoem: mar. 2016.

KATTAN, G. H. et al. Distribution and population density of the endangered cauca guan Pelenope perspicax. Bird Conservation International, Cambridge, v. 16, n. 4, p. 299-307, 2006.

KATTAN, G. H.; MUÑOZ, M. C.; KIKUCHI, D. W. Population densities of curassows, guans and chachalacas (Cracidae): effects of body size, habitat, season, and hunting. The Condor: Ornithological Applications, Washington, v. 118, p. 24-32, 2016.

LANDAU, E. C.; HIRSCH, C.; MUSINSKY, J. Cobertura vegetal e uso do solo do sul da Bahia-Brasil. In PRADO, P. I. et al. (Ed.). Corredor de Biodiversidade da Mata atlântica do sul da Bahia. Ilhéus: IESB; DI; CABS; UFMF; UNICAMP, 2003. CD-ROM.

LAURANCE, W. F. Edge effects in tropical forest fragments: application of a model for the design of nature reserves. Biological Conservation, Boston, v. 57, p. 205-219, 1991.

LAURANCE, W. F.; YENSEN, E. Predicting the impacts of edge effects in fragmented habitats. Biological Conservation, Boston, v. 55, p. 77-92, 1991.

LUNA-MAIRA, L. et al. Habitat use and ecology of wattled curassows on islands in the lower Caquetá River, Colombia. Journal of Field Ornithology, New Ipswich, v. 84, n. 1, p. 23-31, 2013.

MARTÍNEZ-MORALES, M. A. Conservation status and habitat preferences of the cozumel curassow. The Condor, Washington, v. 101, p. 14-20, 1999.

MARTINS, U. R.; GALlILEO, M. H. M. Cerambycidae (Coleoptera) da Serra Bonita, Camacan, Bahia, Brasil. Papéis Avulsos de Zoologia, São Paulo, v. 50, n. 28, p. 435-443, 2010.

MIKICH, S. B. A dieta frugívora de Penelope superciliaris (Cracidae) em remanescentes de floresta estacional semidecidual no centro-oeste do Paraná, Brasil e a relação com Euterpes edulis (Arecaceae). Ararajuba, São Paulo, v. 10, n. 2, p. 207-217, 2002.

PARDINI, R. et al. Beyond the fragmentation threshold hypothesis: regime shifts in biodiversity across fragmented landscapes. Plos One, [s. 1.], v. 5, p. 1-10, 2010.

PAST. Paleontological Statistic (version 3. 10). 2013. Disponível em: <http://palaeo-electronica. org/2001_1/past/issue1_01.htm>. Acesso em: nov. 2016.

PEREIRA, H. M.; DAILY, G. C.; ROUGHGARDEN, J. A framework for assessing the relative vulnerability of species to land-use change. Ecological Applications, Tempe, v. 14, n. 3, p. 730-742, 2004.

PERES, C. A. Effects of subsistence hunting on vertebrate community structure in amazonian forests. Conservation Biology, Cambridge, v. 14, n. 1, p. 240-253, 2000.

PERES, C. A.; PALACIOS, E. Basin-wide effects of game harvest on vertebrate population densities in amazonian forests: implications for animal-mediated seed dispersal. Biotropica, Washington, v. 39, n. 3, 
p. 304-315, 2007.

PULliAM, H. R. Sources, sinks, and population regulation. The American Naturalist, Chicago, v. 32, p. 652-661, 1988.

RIBEIRO, M. C. et al. The Brazilian Atlantic forest: how much is left, and how is the remaining forest distributed? Implications for conservation. BiologicalConservation, Boston, v. 142, p. 1141-1153, 2009.

ROCHA, J. S. Abundância de aves e mamíferos de médio e grande porte de um fragmento de Mata Atlântica na bacia hidrográfica do rio Una. 2014. 66 f. Monografia (Bacharelado em Ciências Biológicas com ênfase em águas continentais) - Universidade Estadual do Sudoeste da Bahia, Jequié, 2014.

ROCHA-SANTOS, L. et al. The shrinkage of a forest: landscape-scale deforestation leading to overall changes in local forest structure. Biological Conservation, Boston, v. 196, p. 1-9, 2016.

SALA, O. E. et al. Global biodiversity scenarios for the year 2100. Science, Washington, v. 287, p. $1770-1774,2000$.

SCARANO, F. R.; CEOTTO, P. Brazilian Atlantic forest: impact, vulnerability, and adaptation climate change. Biodiversity and Conservation, London, v. 24, p. 2319-2331, 2015.

SICK, H.Ornitologia brasileira. Rio de Janeiro: Nova Fronteira, 1997. 912 p.

SILVEIRA, L. F.; SOARES, E. S.; BIANCHI, C. A. Plano de ação para conservação de Galliformes ameaçados de extinção (aracuãs, jacus, jacutingas, mutuns e urus). 6. ed. Brasília: ICMBio; MMA, 2008. 90 p. (Série espécies ameaçadas).

SOUZA, A. S. et al. Estimativas populacionais de mutum-do-sudeste Crax blumenbachii (Aves Cracidae) no Parque Estadual Serra do Conduru, BA, Brasil. In: CONGRESSO DE ECOLOGIA DO BRASIL, 11., 2013, Porto Seguro. Anais... Porto Seguro: Sociedade de Ecologia do Brasil, 2013. p. 1-3.

SRBEK-ARAUJO, A. C.; SILVEIRA, L. F.; CHIARELLO, A. G. The red-billed curassow (Crax blumenbachii), social organization and daily activity patterns. The Wilson Journal of Ornithology, Lawrence, v. 124, n. 2, p. 321-327, 2012.

TABARELLI, M. et al. Prospects for biodiversity conservation in the Atlantic forest: lessons from aging human-modified landscapes. Biological Conservation, Boston, v. 143, p. 2328-2340, 2010.

TABARELLI, M.; LOPES, A. V.; PERES, C. A. Edge-effects drive tropical forest fragments towards an early-sucessional system. The Journal of Tropical Biology and Conservation, [s. 1.], v. 40, n. 6, p. 657661, 2008.

THIOLLAY, J. M. Influence of selective logging on bird species diversity in a Guianan rain forest. Conservation Biology, Cambridge, v. 6, p. 47-63, 1992.

THORNTON, D. H.; BRANCH, L. C.; SUNQUIST, M. E. Response of large galliforms and tinamous (Cracidae, Phasianidae, Tinamidae) to habitat loss and fragmentation in northern Guatemala. Oryx, Cambridge, v. 46, p. 567-576, 2012.

URQUIZA-HAAS, T.; PERES, C. A.; DOLMAN, P. M. Regional scale effects of human density and forest disturbance on large-bodied vertebrates throughout the Yucatán Penisula, Mexico. Biological Conservation, Boston, v. 142, p. 134-148, 2009.

VANTHOMME, $H$. et al. Distribution of a community of mammals in relation to roads and other human disturbances in Gabon, Central Africa. Conservation Biology, Cambridge, v. 27, n. 2, p. 281-291, 2013.

VERACEL. Plano de manejo Reserva Particular do Patrimônio Natural Estação Veracel. [s. 1.]: Veracel e Conservação Internacional, 2007. Disponível em: <http://www.veracel.com.br/rppn-estacao-vercel/plano-de-manejo/>. Acesso em: mar. 2016.

VEC ENGENHEIROS CONSULTORES. Área de proteção ambiental Itacaré/Serra Grande, plano de manejo, zoneamento ecológico-econômico e plano de gestão. 1998. Disponível em: <http://www.inema. ba.gov.br/gestao-2/unidades-de-conservacao/apa/apa-costa-de-itacare-serra-grande/plano-itacare-serra-grande/>. Acesso em: mar. 2017.

WIKIPARQUES. [Website]. 2016. Disponível em: <www.wikiparques.org>. Acesso em: mar. 2016. 